\title{
First-principles calculations of elastic and optical properties of Aluminum Nitride (AIN) in cubic and hexagonal phase
}

\author{
Burhan Ahmed, B. Indrajit Sharma* \\ Dept. of Physics, Assam University, Silchar-788011 \\ *Corresponding Author: indraofficial@rediffmail.com
}

\begin{abstract}
Investigation of elastic and optical properties of AlN in rock salt, zinc blende, and wurtzite phase is done under the framework of Density Functional Theory (DFT) with modified Becke Johnson Generalised Gradient Approximation (mBJ-GGA) as exchange-correlation functional. Elastic properties conclude the bonding nature of the AlN in the rock salt phase is covalent and stiffest, while the bonding nature in the zinc blende and wurtzite phase is found to be ionic and less stiff. The ratio of bulk modulus to shear modulus indicates AIN is brittle in all three phases. The calculated Debye temperature in all three phases is in good agreement with the available theoretical and experimental works. The optical properties calculation shows the AlN is transparent in the low energy range and it has the metallic behavior in the energy range $7.5 \mathrm{eV}$ to $10 \mathrm{eV}$. At the same time, the compound loses its transparency at the high energy range. Our calculated value of the refractive index of AIN in the rock salt, zinc blende, and wurtzite phases is in good agreement with the available experimental and theoretical works.
\end{abstract}

Keywords: Debye temperature; DFT; elastic properties; optical properties.

\section{Introduction}

In recent days III-V semiconductors are showing very much importance in the field of research. Aluminum Nitride (AIN) is the compound that is trending nowadays in the field of science and technology due to its physical properties and potential applications Beshkova, \& Yakimova (2020). The wide bandgap is the main reason for which the semiconductor is studied at a large scale Beiranvand \& Valedbagi (2016). The wide bandgap of the compound raises its application in many electronic, optoelectronic devices, cutting, and machining tool industries Keçik et al., (2015). The high resistivity of the material is an essential factor for the application of its microwave and other optical devices. The electrical and chemical properties of-AlN make it suitable for the use of the compound as a magneto-hydrodynamic (MHD) Kozlovskiy et al., (2019). The AlN compound is generally exhibited in three different crystal structures. The first crystal structure of this ceramic material is obtained and reported by Heinrich Otto in the year 1924. The new structure has the wurtzite form and can be transformed into other phases under suitable pressure. The most stable phase is the wurtzite phase while the metastable and less stable phases are the zinc blende and rock salt phase, respectively. The electronic bandgap of the compound is maximum corresponding to the stable phase and is $6.20 \mathrm{eV} \mathrm{Saib} \mathrm{\&} \mathrm{Bouarissa}$ (2006). In the present work, the elastic and optical parameters of AlN in the three-rock salt, zinc blende, wurtzite phases are calculated using the first principles DFT method. 


\section{Computational Details}

The detailed calculations done in the present work are by using computational analysis. We verified all the results obtained along with experimental and some other theoretical works. The computational tool used for the calculation of elastic constant is the IRelast package developed by M. Jamal with the help of wien2k code. The modified Becke Jhonson potential Generalized Gradient Approximation (mBJ-GGA) and Perdew-Burke-Ernzerhof generalized gradient approximation (PBE-GGA) are used as exchange-correlation potential for the self-consistency field calculation. The elastic and optical calculations are done by taking the equilibrium structural parameters obtained by optimizing the volume of the structure as a function of energy and then fitting into the Birch-Murnaghan Equation of state. The atomic positions for the rock salt are $\mathrm{Al}(0,0,0), \mathrm{N}(0.5,0.5,0.5)$ and for the zinc blende phase are $\mathrm{Al}(0,0,0)$, and $\mathrm{N}(0.25$, $0.25,0.25)$. The wurtzite phase has the atomic positions of $A 1$ at $(2 / 3,1 / 3,0)$ and $(1 / 32 / 31 / 2)$ and $\mathrm{N}$ atom at $(2 / 3,1 / 3, u)$ and $(1 / 32 / 31 / 2+u)$. The parameter $u$ is the internal parameter that measures the separation between $\mathrm{Al}$ and $\mathrm{N}$ atoms along the $c$-axis. There are 48, 24, and 12 symmetry operations are found corresponding to the rock salt, zinc blende, and wurtzite phase. The $R M T K_{\max }$ used for all three phases is 7.0 , where the convergence occurs. The $\mathrm{K}_{\max }$ is the plane wave cut-off, and RMT's are the atomic sphere radii known as Muffin Tin radius. The increase of RMT causes overlapping of atoms and hence causes leaking of electrons. The muffin tin radius, space group, and equilibrium lattice parameters used in this calculation are listed in Table1. The lattice parameters for the wurtzite crystal satisfy the c/a ratio. The number of $k$-points used in this calculation is 5000 for all three phases while the generated $k$-points are 256,165 , and 264 for the rock salt, zinc blende, and wurtzite phase respectively.

Table 1. Different structural parameters were used in this work.

\begin{tabular}{lllll}
\hline & Space group & $R_{\mathrm{MT}}$ & $a(\AA)$ & $c(\AA)$ \\
\hline Rock salt & $225-\mathrm{Fm}-3 \mathrm{~m}$ & Al 1.96 & 4.06 & \\
& & $\mathrm{~N} 1.87$ & & \\
Zinc blende & 216-F-43m & Al 1.75 & 4.37 & \\
& & N 1.84 & & 4.98 \\
Wurtzite & 186-p63mc & Al 1.73 & 3.11 & \\
& & N 1.64 & & \\
\hline
\end{tabular}

\section{Results and Discussion}

\subsection{Elastic properties}

Elastic constants are very essential parameters for describing the properties of a material. There are two suitable methods by which one can derive different ECs. One is the energy approach explained by Stadler where elastic constants are calculated from the ground state energy and the other approach called stress theorem is developed by Nielsen and Martin in which stressstrain relation is used for estimating the elastic constants. Here in this work, we will follow the energy approach by Stadler to evaluate the different elastic constants and hence investigate the 
different elastic properties of the material. The Taylor expansion of total energy $E(V, \delta)$ in respect to a small strain $(\delta)$ is used for defining the elastic constant for the lattice system. Considering the Bravais Lattice vectors of crystal as a matrix form $(R)$, distortion of the lattice $\left(R^{\prime}\right)$ is expressed by multiplying $R$ with a corresponding symmetric distortion matrix, that is $R^{\prime}=R^{*} D$, which can be written in Voigt notation as,

$$
D=\left(\begin{array}{ccc}
1+\delta_{1} & \frac{\delta_{6}}{2} & \frac{\delta_{5}}{2} \\
\frac{\delta_{6}}{2} & 1+\delta_{2} & \frac{\delta_{4}}{2} \\
\frac{\delta_{5}}{2} & \frac{\delta_{4}}{2} & 1+\delta_{3}
\end{array}\right)
$$

By Taylor expansion, the energy of the strained system in terms of the distortion parameters is written as,

$$
\mathrm{E}(\mathrm{V}, \delta)=\mathrm{E}\left(\mathrm{V}_{0}, 0\right)+\mathrm{V}_{0}\left(\sum_{\mathrm{i}=1}^{6} \tau_{\mathrm{i}} \delta_{\mathrm{i}}+\frac{1}{2} \sum_{\mathrm{i}=1}^{6} \sum_{\mathrm{j}=1}^{6} \mathrm{C}_{\mathrm{ij}} \delta_{\mathrm{i}} \delta_{\mathrm{j}}+0\left(\delta^{3}\right)\right)
$$

Here, $V_{\mathrm{O}}$ is the volume of the unstrained crystal system, and if there is no change in volume due to strain called volume conserving technique, the linear term in the above expression will vanish. The parameters $\tau_{i}$ are connected to the linear strain term only and thus no effect in the volume conserving calculation. $\mathrm{V}_{0}$ gives the volume of unstrained crystal system and elastic constants $\mathrm{C}_{\mathrm{ij}}$ are obtained by taking the second-order derivatives of the energy expression,

$$
\mathrm{C}_{\mathrm{ij}}=\frac{1}{\mathrm{~V}_{0}}\left[\frac{\partial^{2} E}{\partial_{\delta_{\mathrm{i}}} \partial_{\delta_{\mathrm{j}}}}\right]_{\delta=0}
$$

Elastic constants determine the stability of the compound. For the cubic crystal, the stability criteria are expressed in terms of three elastic constants,

$$
\begin{gathered}
C_{11}-\left|C_{12}\right|>0 \\
C_{11}+2 C_{12}>0 \\
C_{44}>0
\end{gathered}
$$

And for the hexagonal system, the Born stability criteria are

$$
\begin{gathered}
C_{44}>0 \\
C_{11}-\left|C_{12}\right|>0 \\
\left(C_{11}+C_{12}\right) C_{33}-2 C_{13}^{2}>0
\end{gathered}
$$




\section{Cubic System}

In a cubic symmetry, there are three independent elastic constants $\mathrm{C}_{11}, \mathrm{C}_{12}$, and $\mathrm{C}_{44}$. Three different strains are required to evaluate the three independent constants. The three distortions are considered as,

$$
\begin{aligned}
& D_{1}=\left(\begin{array}{ccc}
1+\delta & 0 & 0 \\
0 & 1-\delta & 0 \\
0 & 0 & \frac{1}{1-\delta^{2}}
\end{array}\right) \\
& D_{2}=\left(\begin{array}{ccc}
1+\delta & 0 & 0 \\
0 & 1+\delta & 0 \\
0 & 0 & 1+\delta
\end{array}\right)
\end{aligned}
$$

And

$$
D_{3}=\left(\begin{array}{ccc}
1 & \delta & 0 \\
\delta & 1 & 0 \\
0 & 0 & \frac{1}{1-\delta^{2}}
\end{array}\right)
$$

$\mathrm{D}_{1}$ gives the volume-conserving orthorhombic distortion while $\mathrm{D}_{2}$ changes the parameters of lattice in an a-lattice direction. Thus $\mathrm{D}_{2}$ changes the volume of distortion lattice, however, the symmetry of the strained lattice system remains the same i.e., cubic. $\mathrm{D}_{3}$ is the volumeconserving monoclinic distortion.

The corresponding energies of the different distortions respectively are calculated as,

$$
\begin{gathered}
\mathrm{E}(\mathrm{V}, \delta)=\mathrm{E}\left(\mathrm{V}_{0}, 0\right)+\mathrm{V}_{0}\left(\left(\mathrm{C}_{11}-\mathrm{C}_{12}\right) \delta^{2}+0\left(\delta^{4}\right)\right) \\
\mathrm{E}(\mathrm{V}, \delta)=\mathrm{E}\left(\mathrm{V}_{0}, 0\right)+\mathrm{V}_{0} \delta\left(\tau_{1}+\tau_{2}+\tau_{3}\right)+\mathrm{V}_{0}\left(\frac{2}{3}\left(\mathrm{C}_{11}+2 \mathrm{C}_{12}\right) \delta^{2}+0\left(\delta^{3}\right)\right) \\
\mathrm{E}(\mathrm{V}, \delta)=\mathrm{E}\left(\mathrm{V}_{0}, 0\right)+\mathrm{V}_{0}\left(\left(2 \mathrm{C}_{44}\right) \delta^{2}+0\left(\delta^{4}\right)\right)
\end{gathered}
$$

Different elastic properties are defined in terms of different elastic constants. For the cubic crystal, different elastic properties are derived from the three elastic constants $\mathrm{C}_{11}, \mathrm{C}_{12}$, and $\mathrm{C}_{44}$. Zener Anisotropy factor $(A)$ can be expressed as,

$$
A=\frac{2 C_{44}}{C_{11}-C_{12}}
$$


The Voigt Bulk modulus $\left(B_{\mathrm{V}}\right)$ and Reuss Bulk modulus $\left(B_{\mathrm{R}}\right)$ of the material can be obtained in terms of the elastic constants $\mathrm{C}_{11}$ and $\mathrm{C}_{12}$ as,

$$
\begin{aligned}
& B_{V}=\frac{C_{11}+2 C_{12}}{3} \\
& B_{R}=\frac{1}{3 S_{11}+6 S_{12}}
\end{aligned}
$$

The Voigt Shear modulus $\left(\mathrm{S}_{\mathrm{V}}\right)$ and Reuss Shear modulus $\left(\mathrm{S}_{\mathrm{R}}\right)$ of the compound are related to the three elastic constants $\mathrm{C}_{11}, \mathrm{C}_{12}$ and $\mathrm{C}_{44}$ as,

$$
\begin{aligned}
S_{V} & =\frac{\left(C_{11}-C_{12}+3 C_{44}\right)}{5} \\
S_{R} & =\frac{15}{4 S_{11}-4 S_{12}+3 S_{44}}
\end{aligned}
$$

Where $S_{\mathrm{ij}}$ is the elastic compliance constants calculated according to Wen et al. (2017).

\section{Hexagonal System}

In hexagonal symmetry, there are five different independent elastic constants $C_{11}, C_{12}, C_{13}, C_{33}$, and $\mathrm{C}_{55}$. Thus five different strains are needed to calculate these constants. The first distortion changes the size of the basal plane keeping the z-axis fixed. Hence the symmetry of the strained lattice remains hexagonal. The first distortion can be written as

$$
\left(\begin{array}{ccc}
1+\delta & 0 & 0 \\
0 & 1+\delta & 0 \\
0 & 0 & 1
\end{array}\right)
$$

The corresponding energy for this distortion can be found as,

$$
\mathrm{E}(\mathrm{V}, \delta)=\mathrm{E}\left(\mathrm{V}_{0}, 0\right)+\mathrm{V}_{0} \delta\left(\tau_{1}+\tau_{2}\right)+\mathrm{V}_{0}\left(\left(\mathrm{C}_{11}+\mathrm{C}_{12}\right) \delta^{2}+0\left(\delta^{3}\right)\right)
$$

Volume conserving distortion is the second type of distortion that produces the orthorhombic symmetry as,

$$
\left(\begin{array}{ccc}
\left(\frac{1+\delta}{1-\delta}\right)^{1 / 2} & 0 & 0 \\
0 & \left(\frac{1-\delta}{1+\delta}\right)^{1 / 2} & 0 \\
0 & 0 & 1
\end{array}\right)
$$


Related energy can be determined from

$$
\mathrm{E}(\mathrm{V}, \delta)=\mathrm{E}\left(\mathrm{V}_{0}, 0\right)+\mathrm{V}_{0}\left(\left(\mathrm{C}_{11}-\mathrm{C}_{12}\right) \delta^{2}+0\left(\delta^{3}\right)\right)
$$

Keeping the symmetry of the strained lattice remains unchanged, the third strain changes the $\mathrm{C}$ lattice parameter. The third distortion is given as

$$
\left(\begin{array}{ccc}
1 & 0 & 0 \\
0 & 1 & 0 \\
0 & 0 & 1+\delta
\end{array}\right)
$$

The energy for the third stain is,

$$
\mathrm{E}(\mathrm{V}, \delta)=\mathrm{E}\left(\mathrm{V}_{0}, 0\right)+\mathrm{V}_{0} \delta\left(\tau_{3}\right)+\mathrm{V}_{0}\left(\frac{\left(\mathrm{C}_{33}\right) \delta^{2}}{2}+0\left(\delta^{3}\right)\right)
$$

The fourth distortion produces the triclinic symmetry of the lattice. The fourth elastic constant $\mathrm{C}_{55}$ is obtained in terms of deformation of the lattice which leads to having an object with low symmetry. This deformation is given by

$$
\left(\begin{array}{lll}
1 & 0 & \delta \\
0 & 1 & 0 \\
\delta & 0 & 1
\end{array}\right)
$$

The energy for this deformation is determined from,

$$
\mathrm{E}(\mathrm{V}, \delta)=\mathrm{E}\left(\mathrm{V}_{0}, 0\right)+\mathrm{V}_{0} \delta\left(\tau_{5}\right)+\mathrm{V}_{0}\left(2 \mathrm{C}_{55}\left(\delta^{2}\right)+0\left(\delta^{3}\right)\right)
$$

The fifth and the last distortion is again the volume conserving which keeps the symmetry of the strained lattice remains hexagonal and it can be written as

$$
\left(\begin{array}{ccc}
(1+\delta)^{-1 / 3} & 0 & 0 \\
0 & (1+\delta)^{-1 / 3} & 0 \\
0 & 0 & \left(1+\delta^{2 / 3}\right)
\end{array}\right)
$$

And energy for the final distortion is

$$
\mathrm{E}(\mathrm{V}, \delta)=\mathrm{E}\left(\mathrm{V}_{0}, 0\right)+\mathrm{V}_{0}\left(\frac{\left(\mathrm{C}_{\mathrm{zz}}\right) \delta^{2}}{9}+0\left(\delta^{3}\right)\right)
$$

Where $\mathrm{C}_{\mathrm{zz}}$ is given by $C_{z z}=C_{11}+C_{12}+2 C_{33}-4 C_{13}$ 
The different elastic properties for the hexagonal systems are defined in terms of different elastic constants. The Voigt Bulk modulus $B_{V}$, Reuss Bulk modulus $B_{R}$ and the Voigt Shear modulus $\mathrm{S}_{\mathrm{V}}$, Reuss Shear modulus $\mathrm{S}_{\mathrm{R}}$ of the crystal are calculated according to Wen et al. (2017). And The Zener Anisotropic factor for the hexagonal system is obtained by

$$
A=\frac{4 C_{55}}{C_{11}+C_{33}-2 C_{13}}
$$

The Hill's average Bulk modulus (B) and shear modulus (S) are given by,

$$
\begin{aligned}
& B=\frac{B_{V}+B_{R}}{2} \\
& S=\frac{S_{V}+S_{R}}{2}
\end{aligned}
$$

Once Bulk modulus and Shear modulus are determined, the Young's $(Y)$ modulus of the material can be found as,

$$
Y=\frac{9 S B}{S+3 B}
$$

Poisson's ratio is obtained by,

$$
v=\frac{3 B-2 S}{6 B+2 S}
$$

Elastic constants are calculated in terms of a deformation matrix (unstrained system) along with an identity matrix, using the FP-LAPW+lo method, which estimates the total energy of the system for the generated structure. By taking the second-order derivative of energy concerning the strained system and dividing it by the volume of the compound and evaluating it to zero strain, gives the number (or liner term) of elastic constants. For the $n$ deformation matrices, $n$ Equations are related to $n$ different elastic constants and thus $n$ number of elastic constants are obtained. For the cubic group, there are mainly three independent elastic constants namely, $\mathrm{C}_{11}, \mathrm{C}_{12}$, and $\mathrm{C}_{44}$ and for the hexagonal groups, there are five different elastic constants namely, $\mathrm{C}_{11}, \mathrm{C}_{12}, \mathrm{C}_{13}, \mathrm{C}_{33}$, and $\mathrm{C}_{55}$. The different elastic constants for the cubic and hexagonal groups are listed in Table 2 and it can be seen that all the independent elastic constants are satisfying the Born stability criteria. The different elastic constants obtained in this work are again compared with the experimental and other theoretical results and are in good agreement. Based on these different elastic constants, different mechanical properties of the solids are studied. The Bulk modulus, which determines the hardness of the material i.e., by how much the solid is resistant to change in its shape, is evaluated by using Equations (17). Along with the bulk modulus, the Shear modulus, which measures the resistance offered against the shear deformation is determined by using Equation (18).In the present work, for the cubic phase (rock salt and zinc blende) and hexagonal phase (wurtzite), the bulk modulus is greater than the shear modulus, which implies that the mechanical stability of the solid is mainly limited by the 
parameter bulk modulus. Another important parameter is Young's modulus which determines the stiffness of the material and is evaluated by using Equation (19). Given the statement, if the material has a larger value of Young's modulus, the material becomes stiffer. In our present work, Young's modulus of rock salt is greater than the wurtzite and then zinc blende phase. Hence the AlN in the rock salt phase is stiffest while the metastable zinc-blende phase is less stiff.

Table 2. The calculated elastic constants of AIN (rock salt, zinc blende, and wurtzite phase).

\begin{tabular}{|c|c|c|c|c|c|c|c|}
\hline $\begin{array}{l}\text { Crystal } \\
\text { Phases } \\
\text { (AlN) }\end{array}$ & & $\mathrm{C}_{11}(\mathrm{GPa})$ & $\mathrm{C}_{12}(\mathrm{GPa})$ & $\begin{array}{l}\mathrm{C}_{13} \\
(\mathrm{GPa})\end{array}$ & $\begin{array}{l}\mathrm{C}_{33} \\
(\mathrm{GPa})\end{array}$ & $\mathrm{C}_{44}(\mathrm{GPa})$ & $\mathrm{C}_{55}(\mathrm{GPa})$ \\
\hline & This work & 354.17 & 200.46 & & & 469.02 & \\
\hline & Expt. values & & & & & & \\
\hline & Theoretical & $423^{a}$ & $167^{\mathrm{a}}$ & & & $306^{\mathrm{a}}$ & \\
\hline & & $396^{\mathrm{b}}$ & $158^{\mathrm{b}}$ & & & $300^{\mathrm{b}}$ & \\
\hline $\begin{array}{l}\text { Rock- } \\
\text { salt }\end{array}$ & & $406^{c}$ & $165^{\mathrm{c}}$ & & & $303^{c}$ & \\
\hline \multirow{9}{*}{$\begin{array}{l}\text { Zinc- } \\
\text { blende }\end{array}$} & This work & 251.80 & 160.23 & & & 213.49 & \\
\hline & Expt. values & & & & & & \\
\hline & \multirow{3}{*}{$\begin{array}{l}\text { Theoretical } \\
\text { values }\end{array}$} & $304^{\mathrm{d}}$ & $160^{\mathrm{d}}$ & & & $135^{f}$ & \\
\hline & & $304^{\mathrm{e}}$ & $168^{\mathrm{f}}$ & & & $199^{\mathrm{e}}$ & \\
\hline & & $348^{\mathrm{f}}$ & $152^{\mathrm{e}}$ & & & $193^{d}$ & \\
\hline & This work & 444.10 & 111.89 & 71.54 & 446.42 & & 119.81 \\
\hline & Expt. values & $394^{g}$ & $134^{\mathrm{g}}$ & $95^{\mathrm{g}}$ & $402^{g}$ & & $121^{\mathrm{g}}$ \\
\hline & \multirow{2}{*}{$\begin{array}{l}\text { Theoretical } \\
\text { values }\end{array}$} & $376^{\mathrm{a}}$ & $127^{\mathrm{a}}$ & $97^{\mathrm{a}}$ & $355^{\mathrm{a}}$ & & $112^{\mathrm{a}}$ \\
\hline & & $398^{\mathrm{h}}$ & $140^{\mathrm{h}}$ & $99^{\mathrm{i}}$ & $389^{\mathrm{i}}$ & & $118^{\mathrm{i}}$ \\
\hline Wurtzite & & $411^{\mathrm{i}}$ & $149^{\mathrm{i}}$ & $127^{\mathrm{h}}$ & $383^{\mathrm{h}}$ & & $96^{\mathrm{h}}$ \\
\hline
\end{tabular}

${ }^{\mathrm{a}}$ Wang et. al., (2010); ${ }^{\mathrm{b}}$ Peng et. al., (2008); ${ }^{\mathrm{c}}$ Kato \& Hama (1994) ; ${ }^{\mathrm{d}}$ Wright et. al., (1997); Kim et. al., (1996); ${ }^{\mathrm{f}}$ Ruiz et. al., (1994); ${ }^{\mathrm{g}}$ Kazan et. al., (2007); ${ }^{\mathrm{h}}$ Wan et. al.,(2001); ${ }^{\mathrm{i}} \mathrm{McNeil}$ et. al., (1993)

The ratio of transverse contraction strain to the longitudinal extension strain in the direction of stretching force, commonly known as Poisson's ratio, is an important elastic parameter of solid determined from elastic constants using Equation (20). Poisson's ratio measures whether the material is ionic or covalent. If the Poisson's ratio is too lower than 0.25 or more, then the compound is said to be a typical covalent compound. On the other hand, if the Poisson's ratio is nearly 0.25 or more, then the compound is said to be a typical ionic compound according to Yildirim \& Koc (2012). In our present work, the Poisson's ratio of metastable zinc-blende and the stable wurtzite phase is found to be nearly 0.25 and that indicates that the zinc blende and wurtzite phase of AIN is ionic. While for the rock salt phase the value of Poisson's ratio is lower than 0.25 and it implies that the rock salt phase is covalent. Another important property 
of the material, whether the behavior of the material is ductile or brittle can be explained in connection with the Bulk modulus and Shear modulus. If the ratio of Bulk modulus to the Shear modulus $(\mathrm{B} / \mathrm{S})$ is greater than 1.75 , then the solid is regarded as ductile otherwise it will be brittle. The present calculation shows the ratio of $\mathrm{B} / \mathrm{S}$ for the rock salt, zinc blende, and wurtzite phase are 1.07, 1.63, and 1.36 respectively. The results show that the material is brittle for all three phases. Another realistic parameter that influences the elastic anisotropy proposed by Zener is called the Zener anisotropy factor. If the Zener anisotropy factor is unity, then the crystal is regarded as isotropic, and while a deviation from unity means the crystal is anisotropic. The Zener anisotropy factor for both the cubical and hexagonal structure of AIN is calculated by using Equation (6) and Equation (16). Our calculation of the elastic anisotropic factor for the rock salt, zinc blende, and wurtzite phase of AlN are found to be $6.1028,4.6633$, and 0.6411 respectively. It concludes wurtzite phase of AIN is more isotropic than the metastable zinc-blende and rock salt phase. The different elastic parameters obtained from different elastic constants are listed in Table 3.

Table 3. Calculated elastic parameters of AIN (rock salt, zinc blende, and wurtzite phase).

\begin{tabular}{l|l|l|l|l|l|l}
\hline $\begin{array}{l}\text { Elastic } \\
\text { parameters }\end{array}$ & \multicolumn{2}{|c|}{ AlN Rock salt } & \multicolumn{2}{c|}{ AlN Zinc blende } & \multicolumn{2}{|c}{ AlN Wurtzite } \\
& Work & $\begin{array}{l}\text { Experimental } \\
\text { and other } \\
\text { reported work }\end{array}$ & $\begin{array}{l}\text { This } \\
\text { Work }\end{array}$ & $\begin{array}{l}\text { Experimental } \\
\text { and other } \\
\text { reported work }\end{array}$ & $\begin{array}{l}\text { This } \\
\text { Work }\end{array}$ & $\begin{array}{l}\text { Experimental } \\
\text { and other } \\
\text { reported work }\end{array}$ \\
\hline A & 6.10 & $0.97^{\mathrm{a}}$ & 4.66 & $2.77^{\mathrm{c}}$ & 0.64 & $0.037^{\mathrm{a}}$ \\
$B_{V}(\mathrm{GPa})$ & 251.69 & & 190.75 & $200^{\mathrm{d}}$ & 204.81 & $201^{\mathrm{i}}$ \\
$B_{R}(\mathrm{GPa})$ & 251.69 & $251^{\mathrm{a}}$ & 190.75 & $216^{\mathrm{e}}$ & 204.95 & $206^{\mathrm{j}}$ \\
$B_{H}(\mathrm{GPa})$ & 251.69 & & 190.75 & $213^{\mathrm{f}}$ & 204.68 & $194^{\mathrm{a}}$ \\
& & & 146.41 & $139.98^{\mathrm{d}}$ & 153.12 & $122^{\mathrm{a}}$ \\
$S_{V}(\mathrm{GPa})$ & 312.15 & $234^{\mathrm{a}}$ & 80.60 & $131^{\mathrm{g}}$ & 147.48 & $121^{\mathrm{a}}$ \\
$S_{R}(\mathrm{GPa})$ & 154.22 & $196^{\mathrm{a}}$ & 116.50 & & 150.30 & $193^{\mathrm{a}}$ \\
$S_{H}(\mathrm{GPa})$ & 233.19 & & 349.75 & $186.11^{\mathrm{d}}$ & 367.77 & $332.14^{\mathrm{k}}$ \\
$Y_{V}(\mathrm{GPa})$ & 662.56 & $587^{\mathrm{b}}$ & 225.65 & $325^{\mathrm{d}}$ & 356.76 & \\
$Y_{R}(\mathrm{GPa})$ & 384.20 & & 290.39 & & 362.29 & \\
$Y_{H}(\mathrm{GPa})$ & 534.50 & & 0.19 & $0.25^{\mathrm{h}}$ & 0.20 & $0.24^{\mathrm{a}}$ \\
$v_{V}$ & 0.06 & $0.16^{\mathrm{a}}$ & 0.30 & & 0.20 & $0.25^{\mathrm{j}}$ \\
$v_{R}$ & 0.24 & $0.16^{\mathrm{b}}$ & 0.25 & & 0.20 & \\
$v_{H}$ & 0.13 & & & & & \\
\hline
\end{tabular}

${ }^{\mathrm{a}}$ Bao et al., (2018); ${ }^{\mathrm{b}}$ Zagorac et al., (2018); ${ }^{\mathrm{C}}$ Benkabou et al., (2003); ${ }^{\mathrm{d}}$ Kumar et al., (2017)

${ }^{\mathrm{e}} \mathrm{Jiao}$ et al., (2011); ${ }^{\mathrm{f} B e c h s t e d t ~}$ et al., (2000); ' $\mathrm{Kanoun} \mathrm{et} \mathrm{al.,} \mathrm{(2004);}{ }^{\mathrm{h}}$ Daoud \& Bouarissa (2019);

${ }^{\mathrm{i}}$ Tsubouchi et al., (1981); ${ }^{\mathrm{j}}$ Liu et al (2017); ${ }^{\mathrm{k}}$ Güler et al., (2014). 
Once the different elastic parameters are found, the different Thermodynamic properties are then calculated in terms of different elastic parameters. The transverse and the longitudinal velocities are calculated by using the following relations,

$$
\begin{gathered}
v_{t}=\left[\frac{S}{\rho}\right]^{\frac{1}{2}} \\
v_{l}=\left[\frac{B+\frac{4 S}{3}}{\rho}\right]^{\frac{1}{2}}
\end{gathered}
$$

Where $\rho$ is the density of the compound. The average sound wave velocity consisting of transverse and longitudinal velocities are then calculated as,

$$
v_{m}=\left[\frac{1}{3}\left(\frac{2}{v_{t}^{3}}+\frac{1}{v_{l}^{3}}\right)\right]^{-1 / 3}
$$

And finally, the Debye temperature $\theta_{\mathrm{D}}$ of the crystal is determined by,

$$
\theta_{D}=\frac{h}{K_{B}}\left[\frac{3 n}{4 \pi}\left(\frac{N_{A} \rho}{M}\right)\right]^{1 / 3} v_{m}
$$

Where $h$ is Plank's constant, $K_{\mathrm{B}}$ is the Boltzmann constant, $N_{\mathrm{A}}$ is the Avogadro's number, $\mathrm{M}$ is the molecular weight, and $n$ is the number of atoms per formula unit. Debye temperature and sound velocities obtained from different elastic constants along with experimental and other

\begin{tabular}{|c|c|c|c|c|c|c|}
\hline \multicolumn{2}{|c|}{ Crystal phases of AlN } & $v_{t}(m / s)$ & $v_{l}(m / s)$ & $v_{m}(m / s)$ & $\theta_{D}$ & $\begin{array}{l}\text { Melting } \\
\text { Temp. (K) }\end{array}$ \\
\hline \multirow[t]{3}{*}{ Rock-salt } & This work & 7627.12 & 11847.10 & 8373.89 & 1222.11 & $2646 \pm 300$ \\
\hline & Expt. values & & & & & \\
\hline & Theoretical values & & & & & \\
\hline \multirow{3}{*}{$\begin{array}{l}\text { Zinc } \\
\text { blende }\end{array}$} & This work & 6049.97 & 10427.50 & 6713.70 & 907.32 & $2041 \pm 300$ \\
\hline & Expt. values & & & & & \\
\hline & Theoretical values & $\begin{array}{l}6127.60^{\mathrm{a}} \\
6186.00^{\mathrm{b}} \\
6165.00^{\mathrm{b}}\end{array}$ & $\begin{array}{l}10658.20^{\mathrm{a}} \\
10576.00^{\mathrm{b}} \\
10483.00^{\mathrm{b}}\end{array}$ & $\begin{array}{l}6805.40^{\mathrm{a}} \\
6859.00^{\mathrm{b}} \\
6833.00^{\mathrm{b}}\end{array}$ & $\begin{array}{l}929.80^{\mathrm{a}} \\
926.00^{\mathrm{b}} \\
888.30^{\mathrm{b}}\end{array}$ & \\
\hline \multirow[t]{3}{*}{ Wurtzite } & This work & 6786.61 .00 & 11143.30 & 7496.50 & 1021.56 & \\
\hline & Expt. values & & & & $1150.00^{c}$ & \\
\hline & Theoretical values & & & & $1025.00^{d}$ & \\
\hline
\end{tabular}
theoretical results are listed in Table 4.

Table 4. Debye temperature and Sound velocities of AlN (rock salt, zinc blende, and wurtzite).

${ }^{\mathrm{a}}$ Daoud \& Bouarissa (2019); ${ }^{\mathrm{b}}$ Zhao-Yong et al., (2010); ${ }^{\mathrm{c}}$ Goldberg et al., (2001); ${ }^{\mathrm{d}}$ Sedmidubský et al., (2001) 
One of the fundamental thermal quantities called Debye temperature is calculated using the relation as given in Equation (24). The transverse, longitudinal, and average sound velocities required in the calculation of Debye temperature are given in Table 4. Debye temperature can explain the different physical properties of solids which are linked to vibrations of lattice Rastogi et al., (2019). From Debye temperature, one can connect other physical properties such as melting temperature. The present calculation shows the Debye temperature corresponding to the metastable phase is $907.2 \mathrm{~K}$ which is slightly lower than the available theoretical value $926 \mathrm{~K}$ and $929.80 \mathrm{~K}$ Zhao et al., (2010). The melting temperature calculated in this work is in good agreement with the other theoretical value and slightly lower than the value $2967.4 \mathrm{~K}$ reported by Daoud \& Bouarissa (2019). Debye temperature of wurtzite phase of AlN calculated at $0 \mathrm{GPa}$ pressure $0 \mathrm{~K}$ temperature is $1021.56 \mathrm{~K}$ which is slightly lower than the experimental value $1150 \mathrm{~K}$ Goldberg et al., (2001). This deviation in the result is because the experiment has done under room temperature and at ambient pressure.

\subsection{Optical properties}

The methodology used for the calculation of optical property is based on the joint density of states modified with the respective dipole matrix elements. The optical properties of solids are due to incoming light which causes a response from the electron system to a time-dependent electromagnetic perturbation. The frequency-dependent complex dielectric function $\varepsilon(\omega)=$ $\varepsilon_{1}(\omega)+i \varepsilon_{2}(\omega)$ containing two dielectric tensor components is directly related to the electron energy band structure of crystals. The optical properties of solids are then characterized by investigating these two dielectric tensor components. This complex dielectric function is the most principal feature to obtain the optical response of solids to electromagnetic interference. The imaginary part of this complex dielectric function is then obtained using Ambrosch-Draxl \& Sofo (2006).

$$
\varepsilon_{2}(\omega)=\left(\frac{4 \pi^{2} e^{2}}{m^{2} \omega^{2}}\right) \sum_{i, j} \int\left\langle i|M| j>^{2} f_{i}\left(1-f_{i}\right) \delta\left(E_{f}-E_{i}-\omega\right) d^{3} k\right.
$$

While the real part of it is extracted from the imaginary part by using the Kramers-Kronig relation Szabo et al., (2010)

$$
\varepsilon_{1}(\omega)=1+\frac{2}{\pi} P \int_{0}^{\infty} \frac{\omega^{\prime} \varepsilon_{2}\left(\omega^{\prime}\right)}{\omega^{\prime 2}-\omega^{2}} d \omega^{\prime}
$$

Where $P$ gives the principal value of the integral.

The real and imaginary part obtained from the above are responsible for providing the basis for the complex refractive index and is defined as,

$$
\tilde{n}(\omega)=n(\omega)+i k(\omega)
$$

$n(\omega)$ and $k(\omega)$ in the above Equation represent the real part and imaginary part of the refractive index, where the imaginary part gives the extinction coefficient. Therefore, the optical parameters of crystalline solids such as refractive index $n(\omega)$, extinction 
coefficients $k(\omega)$, reflectivity $R(\omega)$, absorption coefficients $\alpha(\omega)$, energy-loss function $L(\omega)$ are determined from the functions $\varepsilon_{1}(\omega)$ and $\varepsilon_{2}(\omega)$.

With the knowledge of $\varepsilon_{1}(\omega)$ and $\varepsilon_{2}(\omega)$, the refractive index of the crystals depending on frequency can be calculated by using the relation,

$$
n(\omega)=\left[\frac{\varepsilon_{1}(\omega)}{2}+\frac{\sqrt{\varepsilon_{1}^{2}(\omega)+\varepsilon_{2}^{2}(\omega)}}{2}\right]^{1 / 2}
$$

And the extinction coefficients,

$$
k(\omega)=\left[-\frac{\varepsilon_{1}(\omega)}{2}+\frac{\sqrt{\varepsilon_{1}^{2}(\omega)+\varepsilon_{2}^{2}(\omega)}}{2}\right]^{1 / 2}
$$

Now in case if the absorbing medium is very weak, then one can assume $k(\omega)$ is small enough and can be written as

$$
\begin{aligned}
n & =\sqrt{\varepsilon} \\
k & =\frac{\varepsilon_{2}}{2 n}
\end{aligned}
$$

The above Equations connects the refractive index with the real part and absorption coefficient $\alpha(\omega)$ with the imaginary part of the complex dielectric function and is not valid for the medium which has a very large absorption coefficient.

The absorption coefficients can be calculated from the formula,

$$
\alpha(\omega)=\sqrt{2} \omega\left[\sqrt{\varepsilon_{1}^{2}(\omega)+\varepsilon_{2}^{2}(\omega)}-\varepsilon_{1}(\omega)\right]^{1 / 2}
$$

By getting the value of refractive index and extinction coefficients, the reflectivity of the material can be found by using the following relation

$$
R(\omega)=\frac{(1-n)^{2}+k^{2}}{(1+n)^{2}+k^{2}}
$$

And the energy loss function, $L(\omega)=\frac{\varepsilon_{2}(\omega)}{\varepsilon_{1}^{2}(\omega)+\varepsilon_{2}^{2}(\omega)}$

The optical properties of solids are important to investigate the application of the compound in many optoelectronic devices. Among them, the key parameter is the dielectric function which explains the response of the material. The transition from the valance band to the conduction band provides optical spectra of the crystal. The different optical properties are calculated by taking TB-mBJ as exchange-correlation functional under the framework of wien $2 \mathrm{k}$. In this present work, we have investigated the optical properties such as dielectric function, energy loss function, reflectivity, refractive index, extinction coefficient, etc. for the compound AlN in the rock salt, zinc blende, and wurtzite phase. The results obtained in this calculation are 
again verified with the experimental and other theoretical available works. Different Figures show the calculation of different optical properties as a function of energy where energy is taken in the units of $\mathrm{eV}$ for all the three phases of the AlN compound, extracted from the complex dielectric function. Figure 1 shows the calculated values of the real part of the complex dielectric function as a function of energy where energy is measured in eV. From our calculation, it is visible that the real part has a maximum peak of 12.60, 5.95, and 8.24 starting from the positive value corresponding to the rock salt, zinc blende, and wurtzite phases with the energy value $5.80 \mathrm{eV}, 6.74 \mathrm{eV}$, and $6.32 \mathrm{eV}$ respectively. After the highest peak, the value of the real part of the dielectric constant for all three phases decreases and attains a negative value. This decrease towards the negative value gives the reflectiveness of the material that shows the metallic behaviour of the crystal. Thus in this energy range where the value of the real part is lower than unity, the crystal can be used as a protection from radiation. The static dielectric constant of AlN corresponding to the rock salt, zinc blende, and wurtzite phases are $5.83,3.10$, and 4.12 respectively at zero pressure and zero temperature. It is seen the static dielectric constant is maximum for rock salt and minimum for zinc blende phase. Our calculated value for the zinc blende phase is slightly different from the experimental value 4.68 Jiao et al., (2011) found at room temperature and ambient pressure.

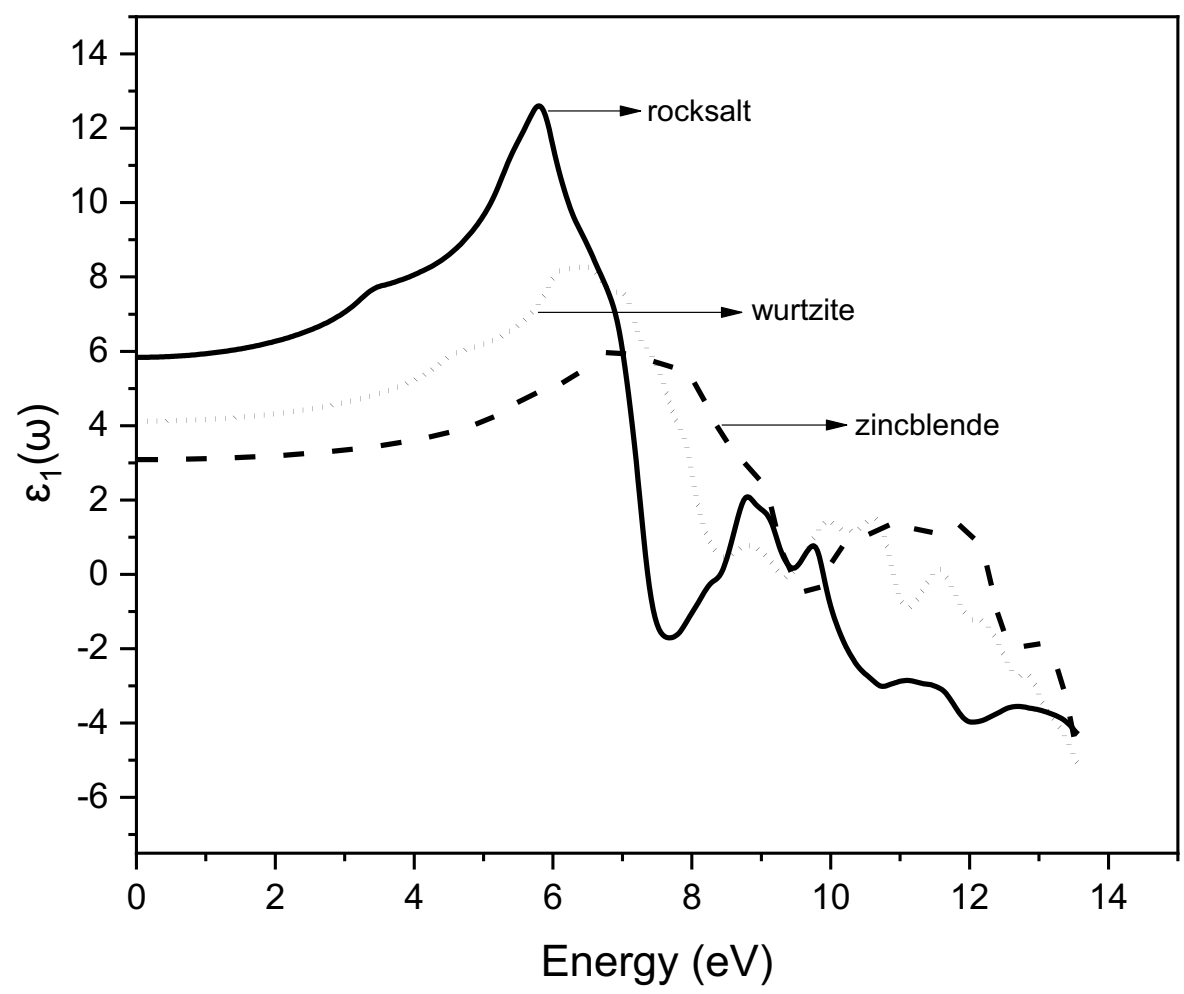

Fig. 1. The real part of the complex dielectric function of AlN (rock salt, zinc blende, wurtzite phase) versus energy. 
Table 5. The static dielectric constant of AIN (rock salt, zinc blende, and wurtzite phase).

\begin{tabular}{llll}
\hline & Rock salt & Zinc blende & Wurtzite \\
\hline This work & 5.83 & 3.10 & 4.12 \\
\hline Other theoretical works & $5.13^{\mathrm{a}}$ & $4.63^{\mathrm{a}}$ & $4.05^{\mathrm{c}}$ \\
& & $4.61^{\mathrm{b}}$ & $4.38^{\mathrm{b}}$ \\
& & $4.46^{\mathrm{c}}$ & $4.39^{\mathrm{a}}$ \\
\hline Experimental & & $4.68^{\mathrm{d}}$ & \\
\hline
\end{tabular}

ajiao et al., (2011); ${ }^{\mathrm{b}}$ Gavrilenko \& Wu (2000); ${ }^{\mathrm{c}}$ Karch \& Bechstedt (1997); ${ }^{\mathrm{d}}$ Akasaki \& Hashimoto (1967).

Figure 2 shows the imaginary part of the complex dielectric function as a function of energy. The threshold energy for the three phases occurs at $3.08 \mathrm{eV}, 5.26 \mathrm{eV}$, and $4.36 \mathrm{eV}$ corresponding to rock salt, zinc blende, and wurtzite phase. Our calculation shows that just after the threshold energy there is a sharp increase of the imaginary part of the dielectric function with the increase of energy and achieved the first peak. The peak in the imaginary part of the dielectric function is maximum in the rock salt phase and minimum in the zinc blend phase. The maximum peak value for the rock salt, zinc blende, and wurtzite phase occurs at 14.59, 6.55, and 8.99 corresponding to the energy $7.19 \mathrm{eV}, 9.15 \mathrm{eV}$, and $8.01 \mathrm{eV}$ respectively.

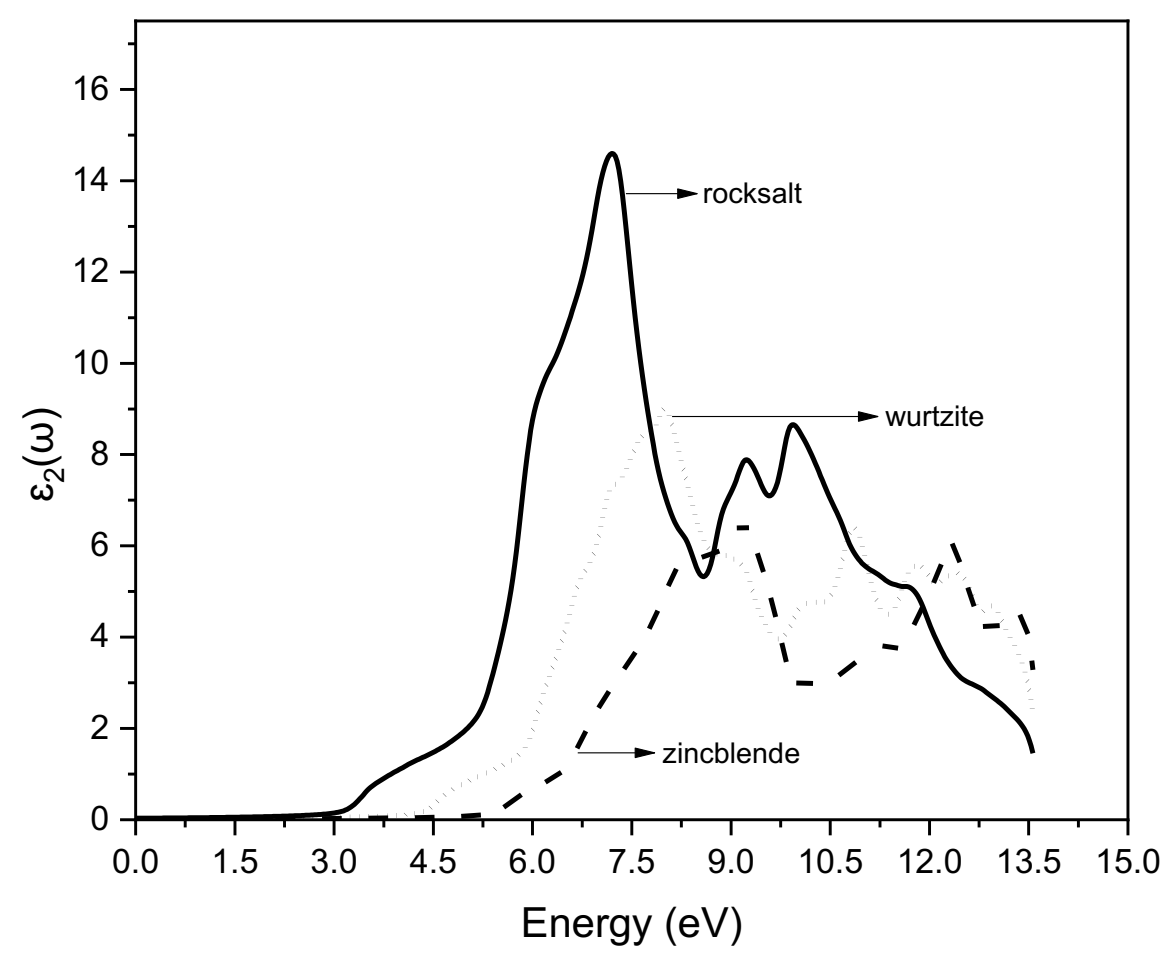

Fig. 2. The imaginary part of the complex dielectric function of AlN (rock salt, zinc blende, wurtzite phase) versus energy. 


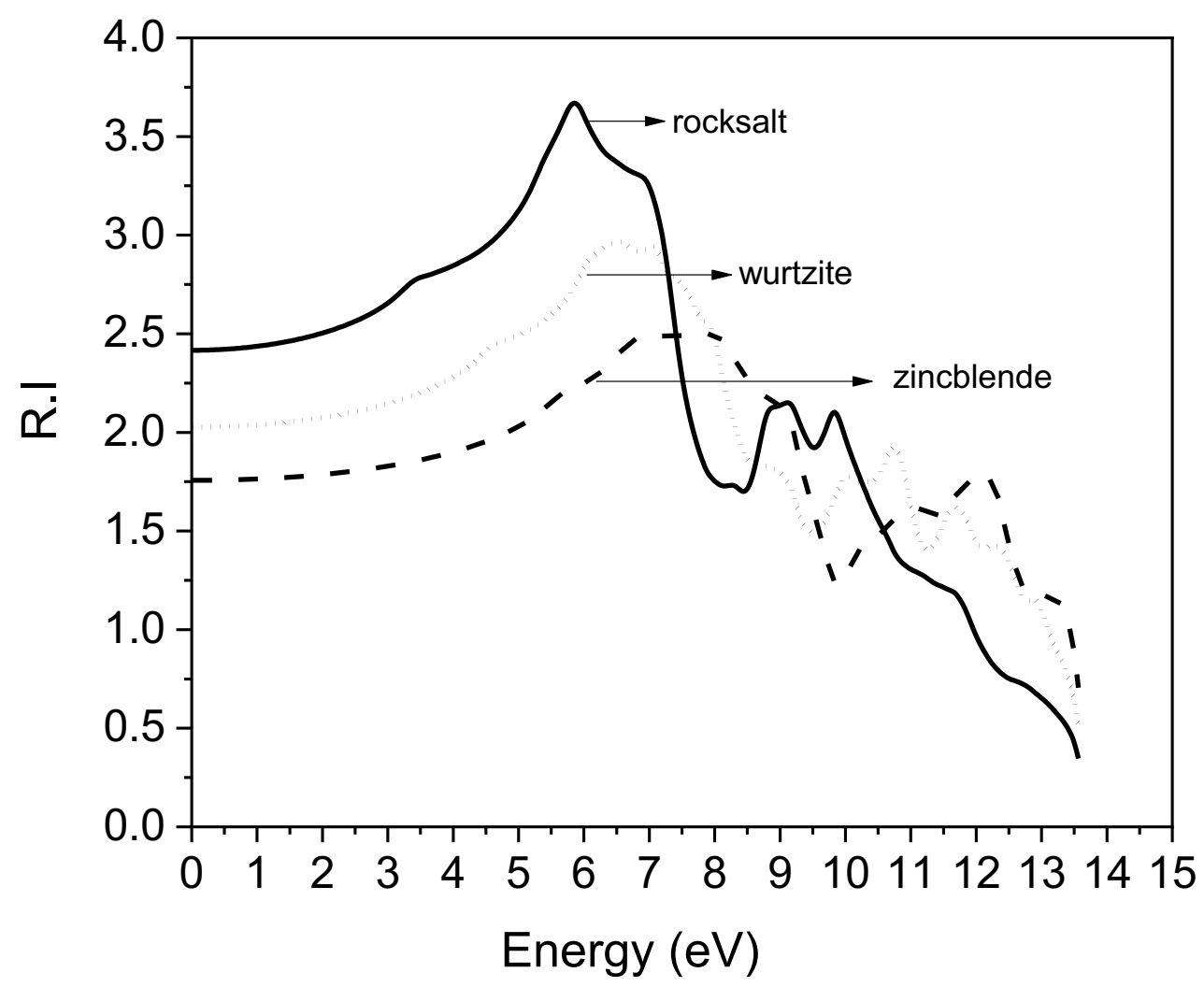

Fig. 3. Refractive Index of AlN (rock salt, zinc blende, wurtzite phase) versus energy.

The refractive index which measures the ratio of the speed of light in a vacuum to any medium of an optical or dielectric medium is calculated using a complex dielectric function as shown in Figure 3. The maximum peak occurs at 3.66, 2.50, and 2.96 corresponding to the rock salt, zinc blende, and wurtzite phase of AlN implies that the rock salt phase has a larger value of the refractive index than that of wurtzite and zinc blende. The metastable phase has the lowest refractive index among all. The same is observed in the case of the real part of the dielectric constant. The energy value corresponding to the maximum peaks are $5.83 \mathrm{eV}, 7.98 \mathrm{eV}$ and $6.51 \mathrm{eV}$ respectively. Our calculated value of the refractive index for the zinc blende phase is 1.75 while that of the experimental value is 2.15 Jiao et al., (2011) at room temperature and ambient pressure. This deviation from the experimental value is due to the present calculation being done at zero pressure and zero Kelvin temperature. The spectrum of refractive index falls at a higher energy value which shows that the material is losing its transparency in the high energy region. The decrease of the refractive index below unity indicates that the velocity of light in a vacuum is less than the phase velocity in the medium. 


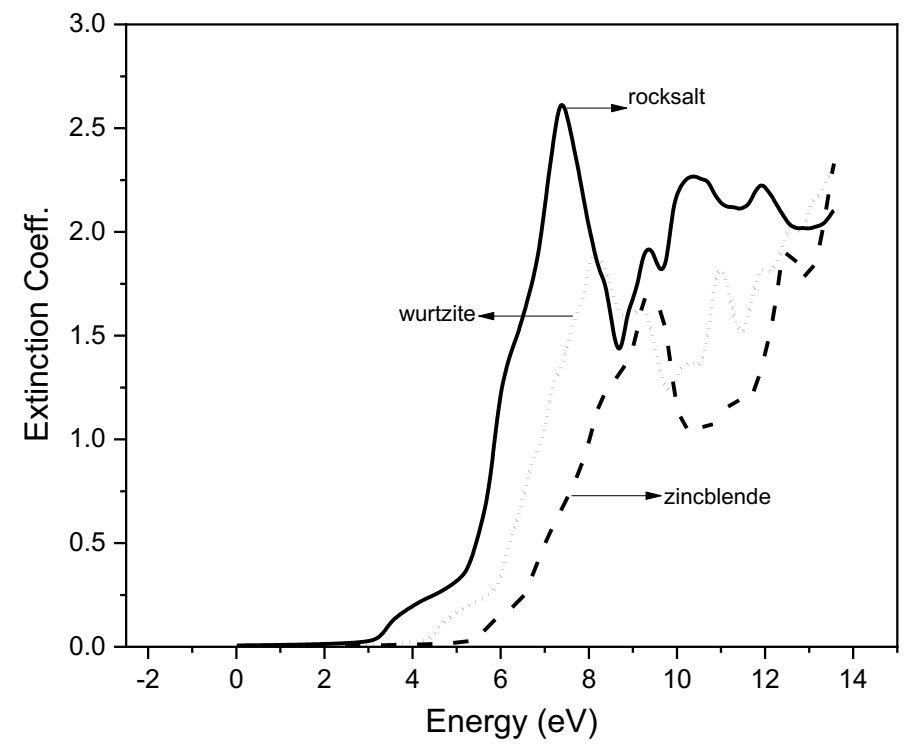

Fig. 4. The extinction coefficient of AlN (rock salt, zinc blende, wurtzite phase) versus energy.

The extinction coefficient measures how strongly a substance can absorb the amount of light incident on it at some specific wavelength. Figure 4 shows the variation of extinction coefficient with the energy for the rock salt, zinc blende, and wurtzite phase of AIN. The threshold energy corresponding to the rock salt, zinc blende, and wurtzite phase occurs at $2.95 \mathrm{eV}, 5.10 \mathrm{eV}$, and $4.01 \mathrm{eV}$ respectively. The first peak of extinction coefficient 2.60 occurs at energy $7.41 \mathrm{eV}$ for the rock salt phase, the extinction coefficient of 1.63 at energy $9.27 \mathrm{eV}$ for zinc blende, and the extinction coefficient of 1.89 at energy $8.14 \mathrm{eV}$ for wurtzite phase of AlN respectively.

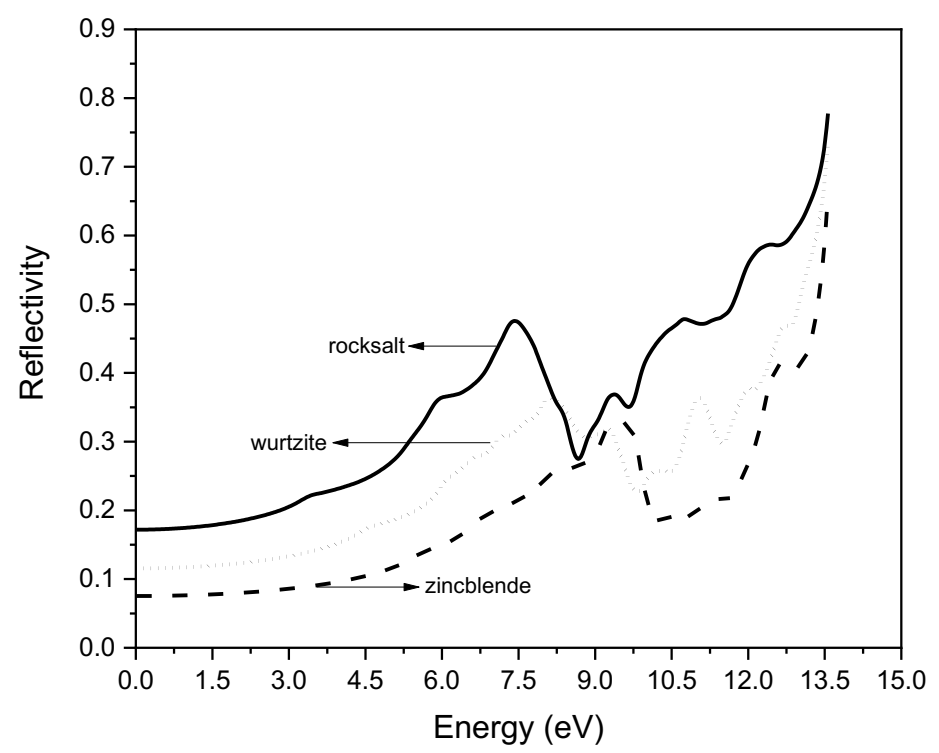

Fig. 5. The reflectivity of AIN (rock salt, zinc blende, wurtzite phase) versus energy. 
The reflection of light from the surface of the crystal is described by reflectivity or coefficient of reflection. Reflectivity spectra of a compound provide the necessary information about complex scattering length density (LSD) of different layers linked with their structural parameters Dhawan et al., (2020). Reflectivity is the measure of energy ratio between incident photons and reflected photons or in another way one can say the reflectivity is the ratio of reflected power to incident power on the surface of the material. Similarly, $\operatorname{transmission}(T)$ is the ratio of transmitted to the incident power. If in the crystal, there is no absorption or scattering of light then from conservation of energy $R+T=1$. Figure 5 shows the reflectivity spectrum of the AIN compound in the three different phases. The reflectivity spectrum starts at $0.17,0.07$, and 0.11 for the rock salt, zinc blend, and wurtzite phase. Figure 5 shows that the reflectivity of the crystal in all three phases is maximum within the energy range of 12 to 14 $\mathrm{eV}$, this is due to the interaction of light and free electrons available in the crystal. There is a sharp drop of reflection coefficient within the range of 7.5 to $10 \mathrm{eV}$ which shows the metallic behavior of the crystal. Our calculation shows that the reflectivity of each phase of the compound is maximum within the energy range of $7.33 \mathrm{eV}$ to $9.75 \mathrm{eV}$. The maximum peaks are observed corresponding to the energy value $7.41 \mathrm{eV}, 9.51 \mathrm{eV}$, and $8.17 \mathrm{eV}$ and the peak values are $0.47,0.32$, and 0.36 for the rock salt, zinc blende, and wurtzite phase of AlN. This gap in peaks can be the result of the interband transition from the valance band to the conduction band. As incident electron losses some discrete amount of energy, the loss spectrum arises due to the excitation of a single electron in the crystal and the excitation of collective oscillation, so-called Plasmon. Figure 6 shows the plot of energy loss function as a function of energy. It is clear from the Figure that threshold energy in rock salt, zinc blende, and wurtzite phase of AIN compound occurs at 3.11, 4.42, and $3.85 \mathrm{eV}$ respectively, before that the curve is flat. This energy gap in the threshold energy is due to the interband transition of an electron at a low frequency. The main peak which is the maximum peak for the three respective phases is located at energy $8.55 \mathrm{eV}, 10.02 \mathrm{eV}$, and $9.61 \mathrm{eV}$, and the peak values are $0.18,0.33$, and 0.24 respectively. The maximum peak is corresponding to the metastable zincblende phase while the minimum peak comes in the less stable rock salt phase of the compound. The energy loss function also depicts that there exist two main peaks corresponding to each phase of the compound. The most prominent peak observed in the energy loss function signifies the characteristics linked with the plasma resonance and the corresponding frequency is called plasma frequency. Above its frequency, the material behaves like a dielectric while below the material behaves like a solid. It is clear from the picture that plasma frequency corresponding to zinc blende phase of AlN is maximum and minimum for its rock salt phase whereas the plasma frequency for its wurtzite phase lies in between them. The absorption coefficient is the absorption of light by an optical medium and is described by the fraction of power absorbed in the unit length of the medium. The absorption spectra of the AlN compound in the three rock salt, zinc blende, and wurtzite phases are shown in Figure 7. It is visible that the crystal has a strong absorptive power when the AlN crystal is in its less stable rock salt phase. This result comes out from the maximum peak of the absorption spectra corresponding to the rock salt phase. The threshold energy occurs at $3.06 \mathrm{eV}, 5.29 \mathrm{eV}$, and $4.36 \mathrm{eV}$ for the rock salt, zinc blende, and wurtzite phase respectively. This indicates that photon having energy greater than 
$3.06 \mathrm{eV}$ will be absorbed by the rock salt phase, while for the case of zinc blende the energy should be greater than $5.29 \mathrm{eV}$, and in the case of the wurtzite phase, the absorption will start at an energy greater than the threshold energy $4.36 \mathrm{eV}$. It concludes below the threshold energy the material behaves like glass or transparent material as the absorption coefficient is very small. The absorption coefficient is small means that transmission is maximum in this region which explains why the material is colorless or behaves like transparent material to the human eye. Hence in this region, the crystal can be used in prisms, optical instruments, and other optical fibre technology.

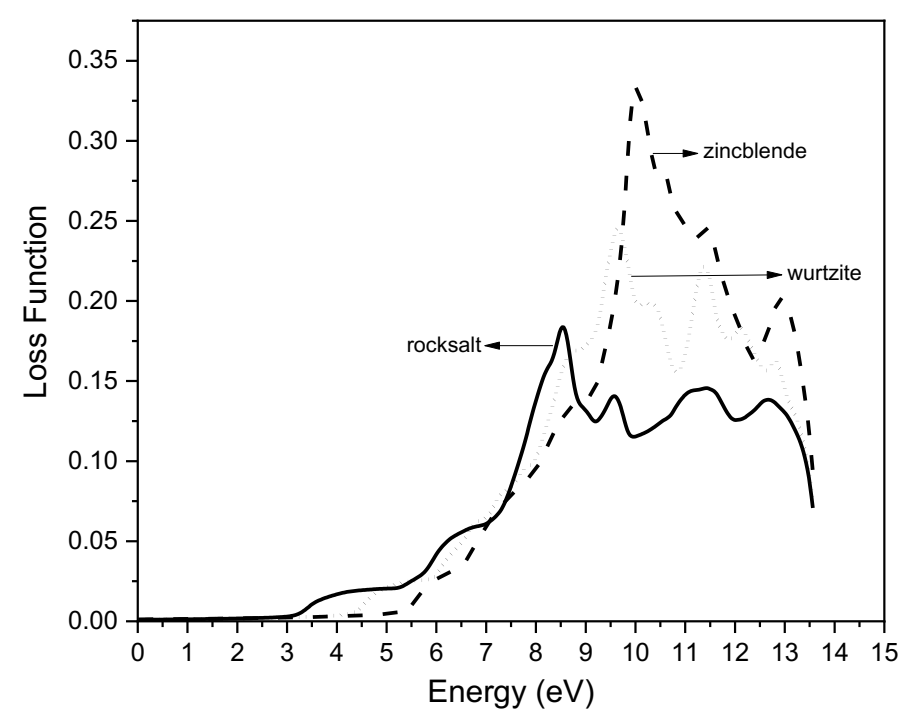

Fig. 6. Loss Function of AIN (rock salt, zinc blende, wurtzite phase) versus energy.

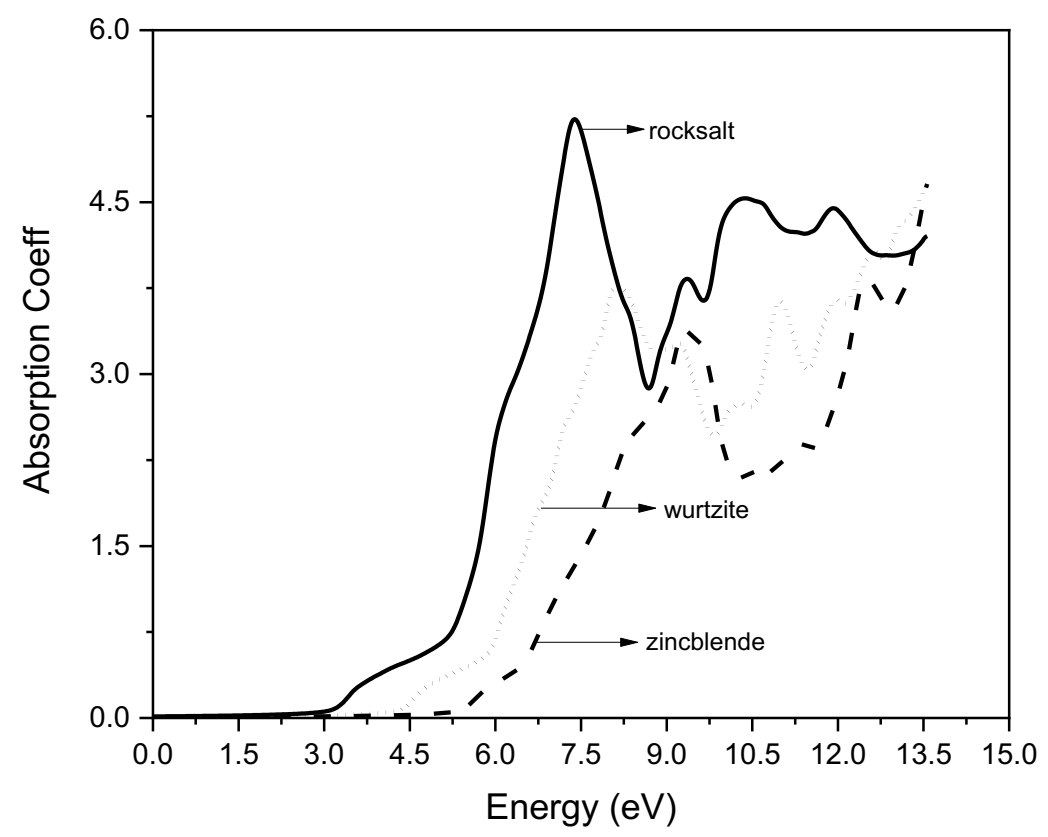

Fig. 7. The absorption coefficient of AlN (rock salt, zinc blende, wurtzite phase) versus energy. 


\section{Conclusions}

The elastic and optical properties of AlN in all the three phases rock salt, zinc blende, and wurtzite have been investigated, and the results obtained are in good agreement with the experimental and available theoretical works. The elastic constants of the three phases of AlN satisfy the Born mechanical stability conditions. The elastic parameter Young's modulus of AlN exhibits its rock salt phase as the stiffest among the three phases. The Poisson's ratio indicates AIN in the zinc blende and the wurtzite phase are ionic whereas its rock salt phase is covalent. Also, the $\mathrm{B} / \mathrm{S}$ ratio shows $\mathrm{AlN}$ is brittle in all three phases and is isotropic in its wurtzite phase than the other phases. The Debye temperature of AIN is in good agreement with the experimental and other theoretical available works. The calculated real part of the dielectric function of AlN in the three phases shows the compound can be used as protection from radiation. The refractive index of AlNis was found to be lowest in the zinc blend phase. However, AlN loses its transparency in the high energy range in all three phases. The reflectivity of the compound AlN indicates it has metallic behavior within the energy range 7.5 $\mathrm{eV}$ to $10 \mathrm{eV}$, and the material behaves transparently below the threshold energy $3.06 \mathrm{eV}, 5.29$ $\mathrm{eV}$, and $4.36 \mathrm{eV}$ for the rock salt, zinc blende, and wurtzite phase respectively.

\section{References}

Akasaki, I., \& Hashimoto, M. (1967). Infrared lattice vibration of vapor-grown AlN. Solid State Communications, 5(11), 851-853.

Ambrosch-Draxl, C., \& Sofo, J. O. (2006). Linear optical properties of solids within the fullpotential linearized augmented plane wave method. Computer physics communications, 175(1), 1-14.

Bao, W., Liu, D., \& Duan, Y. (2018). The first-principles prediction of anisotropic elasticity and thermal properties of potential superhard WB3. Ceramics International, 44(12), 14053 14062.

Bechstedt, F., Grossner, U., \& Furthmüller, J. (2000). Dynamics and polarization of groupIII nitride lattices: A first-principles study. Physical Review B, 62(12), 8003.

Beiranvand, R., \& Valedbagi, S. (2016). Electronic and optical properties of advanced semiconductor materials: BN, AlN, and GaNnanosheets from first principles. Optik, 127(3), $1553-1560$.

Benkabou, F., Certier, M., \& Aourag, H. (2003). Elastic Properties of Zinc-blende Ga N, A $1 \mathrm{~N}$ and In N from Molecular Dynamics. Molecular simulation, 29(3), 201-209.

Beshkova, M., \&Yakimova, R. (2020). Properties and potential applications of two dimensional AlN. Vacuum, 176, 109231. 
Daoud, S., \& Bouarissa, N. (2019). Elastic, piezoelectric, and thermal properties of zincblende AlN under pressure. Theoretical Chemistry Accounts, 138(4), 49.

Dhawan, R., Gupta, R. K., Yadav, P. K., \& Modi, M. H. (2020). Interface asymmetry in AlN/Ni and Ni/AlN interfaces: A study using resonant soft X-ray reflectivity. Applied Surface Science, 529, 147199.1892.

Gavrilenko, V. I., \& Wu, R. Q. (2000). Linear and nonlinear optical properties of group-III nitrides. Physical Review B, 61(4), 2632.

Goldberg Yu. (2001). Properties of Advanced SemiconductorMaterialsGaN, AlN, InN, BN, $\mathrm{SiC}$, SiGe. Eds. Levinshtein M.E., Rumyantsev S.L., Shur M.S., John Wiley \& Sons, Inc., New York, 31-47.

Güler, E., \& Güler, M. (2014). High pressure elastic properties of wurtzite aluminum nitrate. Chinese Journal of Physics, 52(5), 1625-1635.

Jiao, Z. Y., Ma, S. H., \& Yang, J. F. (2011). A comparison of the electronic and optical properties of zinc-blende, rock salt, and wurtzite AlN: A DFT study. Solid-State Sciences, 13(2), 331-336.

Kanoun, M. B., Goumri-Said, S., Merad, A. E., Merad, G., Cibert, J., \& Aourag, H. (2004). Zinc-blende $\mathrm{AlN}$ and $\mathrm{GaN}$ under pressure: structural, electronic, elastic, and piezoelectric properties. Semiconductor science and technology, 19(11), 1220.

Karch, K., \& Bechstedt, F. (1997).Ab initio lattice dynamics of BN and AlN: Covalent versus ionic forces. Physical Review B, 56(12), 7404.

Kato, R., \& Hama, J. (1994). First-principles calculation of the elastic stiffness tensor of aluminum nitride under high pressure. Journal of Physics: Condensed Matter, 6(38),

Kazan, M., Moussaed, E., Nader, R., \& Masri, P. (2007). Elastic constants of aluminum nitride. Physica status solidi c, 4(1), 204-207.7617.

Keçik, D., Bacaksiz, C., Senger, R. T., \& Durgun, E. (2015). Layer-and strain-dependent optoelectronic properties of hexagonal AIN. Physical Review B, 92(16), 165408.

Kim, K., Lambrecht, W. R., \& Segall, B. (1996). Elastic constants and related properties of tetrahedrally bonded BN, AlN, GaN, and InN. Physical Review B, 53(24), 16310.

Kozlovskiy, A., Kenzhina, I., Alyamova, Z. A., \& Zdorovets, M. (2019). Optical and structural properties of AIN ceramics irradiated with heavy ions. Optical Materials, 91, 130137. 
Kumar, V., Singh, B. P., \& Chandra, S. (2017). Optical, elastic, and thermal properties of ZB-AlN semiconductor from first-principle calculations. Indian Journal of Physics, 91(4), 445-451.

Liu, Z., Li, D., Wei, S., Wang, W., Tian, F., Bao, K., \& Cui, T. (2017). Bonding properties of aluminum nitride at high pressure. Inorganic Chemistry, 56(13), 7494-7500.

McNeil, L. E., Grimsditch, M., \& French, R. H. (1993). Vibrational spectroscopy of aluminum nitride. Journal of the American Ceramic Society, 76(5), 1132-1136.

Peng, F., Chen, D., Fu, H., \& Cheng, X. (2008). The phase transition and the elastic and thermodynamic properties of AlN: First principles. Physica B: Condensed Matter, 403(23-24), 4259-4263.

Rastogi, A., Rajpoot, P., \& Verma, U. P. (2019). Properties of group III-V semiconductor: BAs. Bulletin of Materials Science, 42(3), 112.

Ruiz, E., Alvarez, S., \& Alemany, P. (1994). Electronic structure and properties of AlN. Physical Review B, 49(11), 7115.

Saib, S., \& Bouarissa, N. (2006). Electronic properties and elastic constants of wurtzite, zincblende, and rock salt AIN. Journal of Physics and Chemistry of Solids, 67(8), 1888-

Sedmidubský, D., Leitner, J., Svoboda, P., Sofer, Z., \&Macháček, J. (2009). Heat capacity and phonon spectra of A IIIN: Experiment and calculation. Journal of thermal analysis and calorimetry, 95(2), 403-407.

Szabo, Z., Park, G. H., Hedge, R., \& Li, E. P. (2010). A unique extraction of metamaterial parameters based on the Kramers-Kronig relationship. IEEE Transactions on Microwave Theory and Techniques, 58(10), 2646-2653.

Tsubouchi, K., Sugai, K., \& Mikoshiba, N. (1981, October). AlN material constants evaluation and SAW properties on AlN/Al 2 O 3 and AlN/Si. In 1981 Ultrasonics Symposium (pp. 375-380). IEEE.

Wan, S. P., Xia, J. B., \& Chang, K. (2001). Effects of piezoelectricity and spontaneous polarization on electronic and optical properties of wurtzite III-V nitride quantum wells. Journal of Applied Physics, 90(12), 6210-6216.

Wang, A. J., Shang, S. L., Du, Y., Kong, Y., Zhang, L. J., Chen, L., ... \& Liu, Z. K. (2010). Structural and elastic properties of cubic and hexagonal TiN and AlN from first-principles calculations. Computational materials science, 48(3), 705-709. 
Wen, Y., Wang, L., Liu, H., \& Song, L. (2017). Ab initio study of the elastic and mechanical properties of B19 TiAl. Crystals, 7(2), 39.

Wright, A. F. (1997). Elastic properties of zinc-blende and wurtzite AlN, GaN, and InN. Journal of Applied Physics, 82(6), 2833-2839.

Yildirim, A., Koc, H., \& Deligoz, E. (2012). First-principles study of the structural, elastic, electronic, optical, and vibrational properties of intermetallic Pd2Ga. Chinese Physics B, 21(3), 037101 .

Zagorac, J., Zagorac, D., Jovanović, D., Luković, J., \& Matović, B. (2018).Ab initio investigations of structural, electronic, and mechanical properties of aluminum nitride at standard and elevated pressures. Journal of Physics and Chemistry of Solids, 122, 94-103.

Zhang, W., Cheng, Y., \& Zhu, J. (2009). CHEN Xiang-Rong. Structural, thermodynamic and electronic properties of zinc-blende AlN from first-principles calculations [J]. Chinese Physics $B, 18(\mathbf{3}), 1207-1214$.

Zhao-Yong, J., Shu-Hong, M., Tian-Xing, W., \& Ji-Fei, Y. (2010). Theoretical investigation of the elastic, electronic, thermodynamic, and optical properties of the zinc-blende structure AlN under high pressure. Molecular Physics, 108(12), 1641-1648.

Submitted: $25 / 11 / 2020$

Revised: $\quad 30 / 11 / 2020$

Accepted: 24/1/2021

DOI: $10.48129 /$ kjs.v49i1.11177 